\title{
SURFACE PHOTOMETRY AND SPECTRAL ANALYSIS OF Mk50: A SEYFERT 1 NUCLEUS IN AN EARLY TYPE GALAXY
}

\author{
M. G. Pastoriza, E. Bica, And Ch. Bonatto \\ Instituto de Física, UFRGS, Porto Alegre, RS, Brasil \\ E. Mediavilla and E. Perez \\ Instituto de Astrofisica de Canarias, 38200 La Laguna, Tenerife, Spain \\ Received 24 January 1991; revised 28 May 1991
}

\begin{abstract}
We analyze Markarian 50 using $V$ and $I$ CCD frames and IPCS and CCD spectra, respectively, obtained in June 1985 (low state of activity) and May 1990 (high state), covering the range 3200 $\AA<\lambda<10100 \AA$. The surface brightness profiles derived from the images indicate that we are dealing with an early type, elliptical or S0 galaxy, which is supported by the spectral analysis of the stellar population. We study the emission properties of the Seyfert 1 nucleus, after subtraction of the stellar population. In the low state we detect a strong, broad $\mathrm{H} \alpha$ with $\mathrm{FWHM} \approx 4500 \mathrm{~km} \mathrm{~s}^{-1}$, whereas $\mathrm{H} \beta$ is stronger than $[\mathrm{O} \mathrm{III}]_{\lambda 5007}$ by a factor $\approx 2$ (in flux). The stellar population is clear by the presence of $\mathrm{Ca}$ II $H$ and $K, G$ band, and $\mathrm{Mg} \mathrm{I}$, and its continuum contribution is more than $92 \%$ for $\lambda>4000 \AA$. In the high state $\mathrm{H} \alpha$ broadens to FWHM $\approx 5400 \mathrm{~km} \mathrm{~s}^{-1}, \mathrm{H} \beta$ becomes a factor $\approx 7$ (in flux) stronger than $[\mathrm{O} \mathrm{III}]_{\lambda 5007}$, and $\mathrm{He}_{\lambda 5876}$ is prominent. The stellar population fractions in the high state continuum are $48 \%$ at $\lambda 3800 \AA, 71 \%$ at $\lambda 5000 \AA$, and $82 \%$ at $\lambda 8900 \AA$. Permitted Fe II emission has similar intensities in the high and low states, showing that its formation zone does not coincide with that of the broad $\mathrm{H}$ I and $\mathrm{He} \mathrm{I}$ lines, i.e., it is farther than five light years from the central source. The off-nuclear spectra centered at $8^{\prime \prime}(\approx 5 \mathrm{kpc}$ ) have [O II], [O III], [N II], $\mathrm{H} \alpha$, and $\mathrm{H} \beta$ at least in part produced by radiation from the strong central source, and the stellar population is dominated by a typical red stronglined bulge population while the contribution of young components (age $<5 \times 10^{8} \mathrm{yr}$ ) is $\approx 7 \%$ at $\lambda 5870 \AA$. The spectral analysis also shows that the point source at $17^{\prime \prime}$ northeast of Mk50 is not a companion, but a foreground star of spectral type K7V-M0V.
\end{abstract}

\section{INTRODUCTION}

Seyfert nuclei occur in a wide range of morphological types, but they are seldom seen in early type galaxies (Adams 1977). They appear quite often in pairs and/or in interacting systems (Dahari 1984). Markarian 50 is a Seyfert 1 nucleus (Sargent 1970; Weedman 1976) with evidence of having a host galaxy of elliptical type with an extended halo elongated in the north-south direction (Adams 1977). A compact object lies at 17 " north-east, but it does not seem to cause isophotal distortions in Mk50 (Dahari 1984). $U, B$, and $V$ photoelectric photometry indicates variability of the Seyfert nucleus (Penfold 1979).

In this paper we report results obtained from spectrophotometry and surface photometry of Mk50. Using this data we study the surface brightness distribution and the nature of the stellar population, as well as the properties of the emitting gas. In particular we infer on the nature of the host galaxy and the nearby point source. In Sec. 2 we describe the observations. In Secs. 3 and 4 we analyze the photometric and spectrophotometric data. In Sec. 5 we discuss the stellarsubtracted emission spectra, and finally the concluding remarks are given in Sec. 6.

\section{OBSERVATIONS}

$V$ and $I$ CCD images were obtained with the $1.0 \mathrm{~m}$ Jacobus Kapteyn Telescope at Roque de Los Muchachos, Canary Islands, Spain, in January 1986. The pixel size at the Cassegrain focus corresponds to 0.414 " with the RCA SID 501 detector. Photometric standard stars from Landolt's list (1983) were observed to flux calibrate the data. A standard reduction procedure was used for the CCD imaging. Spec- trophotometric observations in the range 3200-7000 $\AA$ with $\approx 4 \AA$ (FWHM) resolution were carried out with the IPCS detector attached to the Cassegrain focus of the $2.5 \mathrm{~m}$ Isaac Newton Telescope at the same observatory on 16 June 1985. The pixel size is $1.8^{\prime \prime}$ and a long slit was set at P.A. $=45^{\circ}$ in order to obtain simultaneously spectra of the galaxy and the stellar object located at $\approx 17^{\prime \prime}$ northeast of the nucleus. The slit width was 2 " and the seeing was comparable. Standard photometric stars were observed in order to flux calibrate the spectra. The nuclear spectrum was obtained summing up the central 4 pixels, and the off-nuclear spectrum corresponds to an average of three pixels on each side of the nucleus centered at $R \approx 8^{\prime \prime}$. Assuming throughout this paper $H_{0}=55 \mathrm{~km} \mathrm{~s}^{-1} \mathrm{Mpc}^{-1}$, the central region covers $0 \leqslant R \leqslant 2.2 \mathrm{kpc}$, and the external region $3.5 \leqslant R \leqslant 6.7 \mathrm{kpc}$. Additional spectra in the range $3200-10100 \AA$ with $\approx 15 \AA$ resolution have been taken at the ESO La Silla $1.5 \mathrm{~m}$ telescope with the high resolution RCA CCD No. 13 attached to the Boller and Chivens spectrograph on 21 May 1990. The slit was set in the east-west direction and extractions were made similarly to those of the IPCS frame.

\section{SURFACE PHOTOMETRY}

\subsection{Luminosity Profiles}

As quoted by Adams (1977) this galaxy could be one of the very few Seyfert nucleus in an elliptical galaxy. Its flux density at $1415 \mathrm{MHz}$ is <4.0 mJy (Wilson \& Meurs 1982) so it cannot be classified as a broad-line radio galaxy (Osterbrock 1988). Mk50 appears in the $V$ and $I$ isophotal maps [Figs. 1(a) and 1(b)] with a main body surrounded by a 


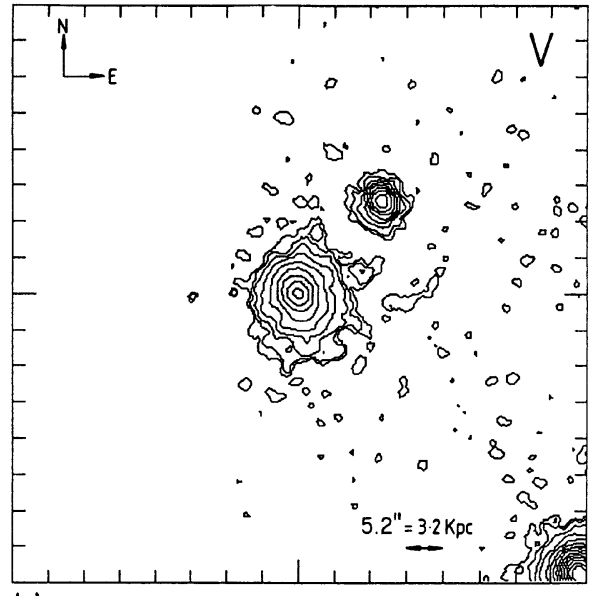

(a)

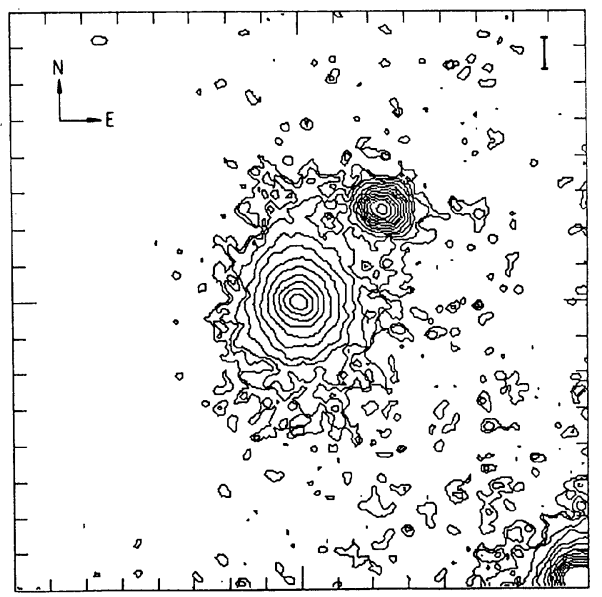

(b)

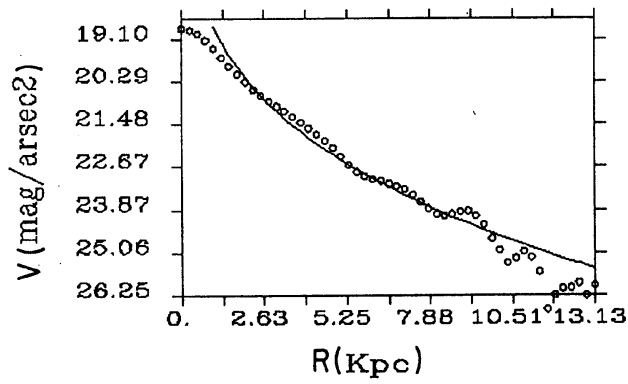

(c)

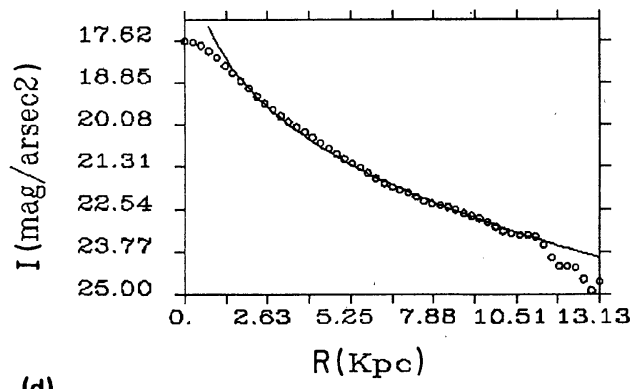

(d)

FIG. 1. (a) and (b) $V$ and $I$ CCD images of Mk50; (c) and (d) the $R^{1 / 4}$ law fitting to the observed profiles. diffuse structure elongated in the north-south direction. The ellipticity $e=0.80$ was measured fitting ellipses to the external isophotes. From the magnitude curve of growth we derive total apparent magnitudes $V=15.1$ and $I=13.8$. The absolute magnitudes $M_{V}=-20.50$ and $M_{I}=-21.84$ and major diameter $D \approx 23 \mathrm{kpc}$ have been calculated using the radial velocity (Sec. 4) $V_{R}=6910 \mathrm{~km} \mathrm{~s}^{-1}$. Radial luminosity profiles were elliptically averaged (Boronson 1981) and a least-squares fit to the averaged $V$ and $I$ profiles was applied using a de Vaucouleurs function $\mu=\mu_{\mathrm{e}}+8.325 \times\left[\left(R / R_{\mathrm{e}}\right)^{1 / 4}-1\right]$ for the bulge and an exponential disk $\mu=\mu_{0}+1.0857 \times \alpha R$ (Freeman 1970). The Bulge range was defined outside the central 3 arcsec in order to avoid the contamination from the central stellarlike source. After several iterations the fitting program found convergence for the following Bulge parameters: effective surface brightness $V_{\mathrm{e}}=19.6, I_{\mathrm{e}}=19.1$ and effective radius, in kpc, $R_{\mathrm{e}}(V)=1.5, R_{\mathrm{e}}(I)=2.1$.

These bulge parameters are comparable to those observed in elliptical and lenticular galaxies (Simien \& de Vaucouleurs 1986; Mediavilla et al. 1989). Fitting the luminosity profiles with a pure exponential disk component proved to be very unsatisfactory, and in Figs. 1(c) and 1(d) we present the luminosity profiles fitted by the $R^{1 / 4}$ law (continuous lines). The averaged $V$ profile is less smooth than that of $I$, presenting small departures from the $R^{1 / 4}$ law, in the form of bumps, which might be caused by recent star formation (see below).

\subsection{Color Index}

The behavior of the color index $(V-I)$ as a function of distance from the center up to $R=8 \mathrm{kpc}$ is illustrated in Fig. 2 , showing a red gradient for the lower envelope with increasing radius, and two conspicuous bumps at $R \approx 3.5$ and $6.5 \mathrm{kpc}$ about $0.2 \mathrm{mag}$ bluer than the underlying red stellar population color. Comparing the $V$ and $I$ maps (Fig. 1) we cannot see knots or spots that would evince the existence of localized bluer regions. So the two bumps represent the averaged color of the stellar population in two particular annuli. The luminosity profiles can be fitted by the $R^{1 / 4}$ law indicating that up to $R \approx 10 \mathrm{kpc}$ the dominant component is a typical bulge. The lower envelope indicates that the underlying

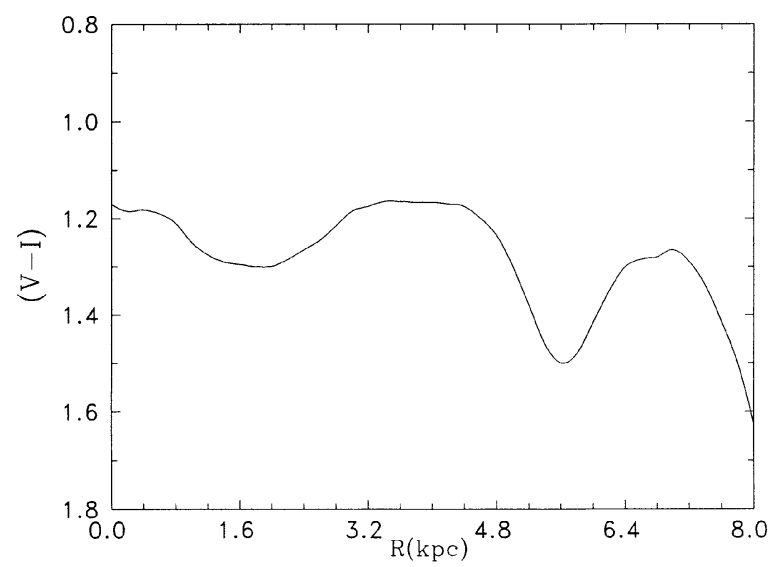

FIG. 2. $(V-I)$ color index. Notice the two blueish bumps superimposed on a red substract. 
population has $(V-I) \approx 1.5$, compatible with the color of one dominated by an old component (age $\approx 10^{10} \mathrm{yr}$ ) with a metallicity $\left(Z / Z_{\odot}\right)=+0.3$ (see Sec. 5). In the same way we can interpret the $(V-I)$ color of the bumps as a mixture of a dominant underlying $E 2$ template spectrum with a contribution of about $1 \%$ in mass ( $\approx 20 \%$ in flux at $\lambda 5870 \AA$ ) of a burst of star formation age-centered at $\approx 10^{8}$ years (Bica $e t$ al. 1990).

\subsection{Interpretation}

The two principal results from the surface photometry are (a) the $V$ and $I$ profiles are well fit by a de Vaucouleurs law, which implies that Mk50 is an elliptical galaxy. However the $V$ scale length $R_{\mathrm{e}}(V)=1.5 \mathrm{kpc}$ results $\approx 25 \%$ smaller than that in $I$. (b) the $(V-I)$ color profile grows redder with radius and has two blue bumps (rings ?) on it. Ellipticals generally grow bluer with radius, whereas Mk50 grows redder. This fact together with the bumps indicates that the star formation history in this galaxy has not been normal. The star formation is strong enough to be detected in the $(V-I)$ color index, but not to destroy the overall de Vaucouleurs profiles. It does disturb the $V$ profile by shortening its scale length. Indeed models of starbursts superimposed on old populations indicate that $V$ flux changes are larger than those in $I$ for the resulting composite population (Bica et al. 1990).

We conclude that Mk50 is an extreme case of elliptical galaxies which has had gas supply for star formation. Even normal ellipticals, when observed in detailed $\mathrm{H} \alpha$ imaging, have revealed the presence of extended gas (Kim 1989).

\section{SPECTROPHOTOMETRY}

The nuclear and off-nuclear spectra were rebinned to the rest frame with $V_{R}=6910 \mathrm{~km} \mathrm{~s}^{-1}$, and have been corrected for a foreground reddening with $\mathrm{E}(B-V)=0.025$, based on data in de Vaucouleurs (1976), Sandage \& Tammann (1981), and Burstein \& Heiles (1984). The nuclear spectra obtained in 1985 and in 1990 are plotted in Fig. 3(a) in which the 1990 spectrum has been displaced three flux units upwards for clarity reasons. In Table 1 we give a selection of continuum points relative to $\lambda 5870 \AA$, which shows striking differences between the two epochs. It is clear that the first spectrum was taken when Mk50 was in a low state of activity, in which the underlying stellar population absorption lines can readily be seen (e.g., $\mathrm{Ca}$ II $H$ and $K, G$ band, $\mathrm{Mg}$ I, and $\mathrm{Na} \mathrm{I}$ ). The 1990 nuclear spectrum represents a high state of activity, in which the broad components of the Balmer lines and the steep rise to the blue are very conspicuous. Stellar population features are completely drowned in the emission. The off-nuclear spectra taken in 1985 and 1990 are very similar, thus we have averaged them out to produce a spectrum with a better signal-to-noise ratio.

In this section we study the stellar population of Mk50 using the 1985 low-state nuclear spectrum and the off-nuclear average spectrum. The emission lines and the spectral variation which occured between 1985 and 1990 will be studied in Sec. 5.

\subsection{Stellar Population}

We analyze the stellar population of the low state nuclear spectrum and the off-nuclear one. The population synthesis method described in Bica (1988, and references therein) is employed. This method uses as base star cluster properties
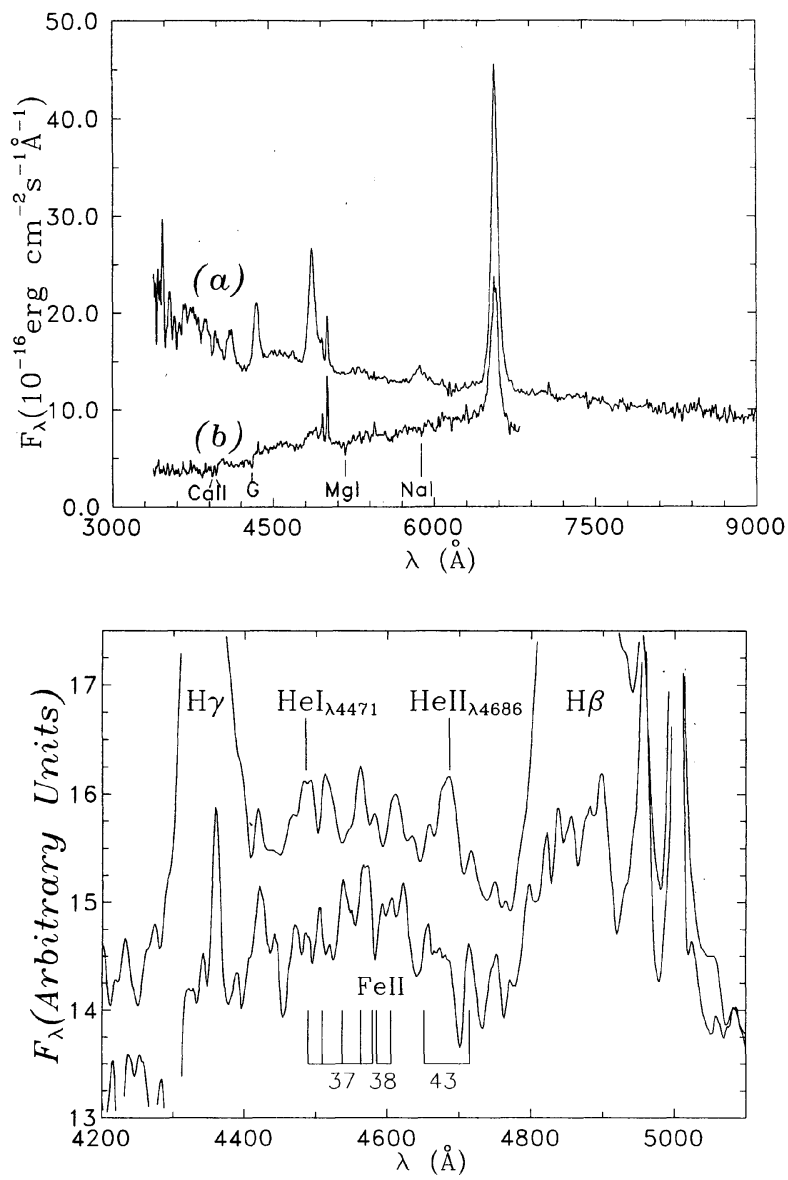

Fig. 3(a). Mk50 nuclear spectra obtained in 1990 (a) and 1985 (b). Absorption features from the stellar population are clearly seen in (b). In (a) the spectrum rises to the blue and the $\mathrm{H}_{\mathrm{I}}$ lines are very conspicuous. The 1990 spectrum has been displaced 3 flux units upwards for clarity reasons. Ordinate units in $10^{-16}$ ergs $\mathrm{cm}^{-2} \mathrm{~s}^{-1} \AA^{-1}$. (b) Fe II lines in the region between $\mathrm{H} \gamma$ and $\mathrm{H} \beta$ have nearly the same intensity in both (high- and lowstate) spectra. Notice that the only significant differences in the overall shape between $4400 \leqslant \lambda \leqslant 4700 \AA$ are due to $\mathrm{He} \mathrm{II}_{\lambda 4686}$ and $\mathrm{He} \mathrm{I}_{\lambda 4471}$. The spectra are plotted in scale as in (a), and a constant was added to the lowstate spectrum for ease of visualization.

rather than those of stars, and results are age/metallicity fractions. Using the same continuum tracing criterium and window limits for equivalent widths, we have measured the main absorption line features, which are shown in Table 2. The synthesis is computed using as base the grid star cluster properties in Bica \& Alloin (1986). The inverse problem is solved by generation of combinations of these elements at $10 \%$ step. Each combination is compared to the observed equivalent widths and if reproduced within error bars, they

TABLE 1. Continuum points relative to $\lambda 5870 \AA$.

\begin{tabular}{cccccccccc}
\hline \hline Spectrum & $3350 \AA$ & $4000 \AA$ & $5000 \AA$ & $5870 \AA$ & $6700 \AA$ & $8000 \AA$ & $9000 \AA$ & $10000 \AA$ \\
\hline Nuclear-Low State & 1.03 & 0.87 & 1.00 & 1.00 & 0.87 & - & - & - \\
Nuclear-High State & 1.69 & 1.27 & 1.14 & 1.00 & 0.92 & 0.74 & 0.62 & 0.55 \\
Off-Nuclear & - & 0.60 & 0.91 & 1.00 & 0.89 & - & - & - \\
\hline \hline \multicolumn{8}{c}{ Notes to TABLE 1 } \\
Continuum absolute values at $\lambda 5870 \AA$ are, respectively $7.54,9.53$, and \\
$1.57 \times 10^{-16} \mathrm{erg} \mathrm{cm}^{-2} \mathrm{~s}^{-1} \AA^{-1}$. The errors are less than $2 \%$.
\end{tabular}


TABLE 2. Equivalent widths for the stellar population synthesis.

\begin{tabular}{ccccccc}
\hline \hline Feature & CaII K & CN band & H $\delta$ & G band & H $\gamma$ & MgI+MgH \\
Window $^{(1)}$ & $3908-3952$ & $4082-4124$ & $4150-4214$ & $4284-4318$ & $4318-4364$ & $5156-5196$ \\
\hline Off-Nuclear & 14.5 & 8.5 & 3.9 & 7.5 & 4.6 & 7.4 \\
Error Bar & 2.0 & 2.0 & 2.0 & 1.2 & 1.5 & 1.5 \\
Nuclear & 8.0 & 11.3 & 6.0 & 7.5 & - & 7.4 \\
Error Bar & 2.5 & 3.5 & 1.2 & 2.2 & - & 1.5 \\
\hline \hline
\end{tabular}

Notes to TABLE 2

Equivalent Widths in $\AA$; Nuclear corresponds to the 1985 low-state spectrum, and Off-Nuclear to the average spectrum of the runs 1985 and 1990; (1) windows used to measure the Equivalent Widths.

are considered to be a possible solution. The error bars for each line are given in Table 2 , and for each analyzed spectrum they indicate the spectral lines which have been effectively used in the respective synthesis. We adopt as a final result the average of all these solutions after a check if the individual ones constitute variations of a single family of results. The typical dispersion of the flux fractions in the average solution is $\approx 10 \%$. The ratio number of solutions with respect to number of generated combinations [see Tables 3 (a) and 3 (b) and discussions below] resulted $\approx 0.5 \%$ in both nuclear and off-nuclear models. Although this is not as "unique" as the syntheses in Bica (1988) it is still very significant. The reason why the same level of "uniqueness" is not reached is because we are dealing with a lower signal-tonoise ratio in the present spectra and furthermore we do not dispose of the near infrared $\mathrm{Ca}$ II triplet owing to emission contamination.

We use chemical enrichment paths as those in Bica (1988), i.e., all the enrichment takes place in the old age bin and the metallicity is assumed to be constant for $t<5 \times 10^{9}$ yr. We have tested different paths with maximum $\left(Z / Z_{\odot}\right)$ attaining, respectively, $-0.5,0.0$ (solar), +0.3 , and +0.6 dex. The solution well for the nuclear and off-nuclear spectra resulted to be +0.3 , although solutions are as well found for solar metallicity, which suggests that the more appropriate "average" maximum metallicity should be around +0.2 dex. Nine elements were used in the calculations, which are shown in Tables 3(a) and 3(b), respectively, for the nuclear and off-nuclear spectra. The nine elements at $10 \%$ step generate 43758 combinations. In the nuclear case we get 240 solutions, and in the off-nuclear one 215 . This means that only $0.55 \%$ and only $0.49 \%$ of the generated combinations

TABLE 3(a). Low-state nuclear spectrum.

\begin{tabular}{cccccccc}
\hline \hline HII R & $10^{7}$ & $10^{8}$ & $5 \times 10^{8}$ & $10^{9}$ & $5 \times 10^{9}$ & $>10^{10}$ & {$\left[\frac{Z}{z_{\odot}}\right]$} \\
\hline 7.9 & 6.7 & 5.0 & 7.6 & 18.0 & 23.2 & 27.1 & +0.3 \\
& & & & & & 2.5 & -1.0 \\
& & & & & & 2.0 & -2.0 \\
\hline \hline
\end{tabular}

Notes to TABLE 3(a)

Combinations : 43758; Solutions : 240

Average line residuals $(\AA): \Delta($ CaII $\mathrm{K})=-2.1, \Delta(\mathrm{CN})=3.2, \Delta(\mathrm{G})=1.7$, $\Delta(\mathrm{MgI}+\mathrm{MgH})=1.0, \Delta(\mathrm{H} \delta)=-0.1$. The results are expressed as percentage flux fractions at $\lambda 5870 \AA$.

\begin{tabular}{ccccccccc}
\multicolumn{8}{c}{ TABLE 3(b). Off-nuclear spectrum. } \\
\hline \hline HII R & $10^{7}$ & $10^{8}$ & $5 \times 10^{8}$ & $10^{9}$ & $5 \times 10^{9}$ & $>10^{10}$ & {$\left[\frac{Z}{Z_{\odot}}\right]$} \\
\hline 1.5 & 3.0 & 0.1 & 1.5 & 8.0 & 37.1 & 23.4 & +0.3 \\
& & & & & & 16.8 & -1.0 \\
& & & & & & 8.6 & -2.0 \\
\hline \hline
\end{tabular}

Notes to TABLE 3(b)

Combinations : 43758; Solutions : 215

Average line residuals $(\AA): \Delta($ CaII $\mathrm{K})=1.1, \Delta(\mathrm{CN})=-1.1, \Delta(\mathrm{G})=0.03$, $\Delta(\mathrm{MgI}+\mathrm{MgH})=0.7, \Delta(\mathrm{H} \delta)=-1.3, \Delta(\mathrm{H} \gamma)=-0.7$. are solutions, which is a safe indication of the uniqueness degree of the result. With respect to the Balmer lines in the nuclear spectrum only $\mathbf{H} \delta$ was used and in the off-nuclear one only $\mathbf{H} \delta$ and $\mathrm{H} \gamma$ were used in the computations, because they were estimated not to be significantly contaminated by emission. The residuals of each line are also indicated in Tables 3 (a) and 3(b). In the computations a featureless continuum from real $\mathbf{H}$ II regions is used as the youngest grid element. It acts as a dilution of absorption features in the computations. In the case of the low state nuclear spectrum it represents in first order a possible mixture of present star formation plus a power-law component. Indeed Table 3(a) shows that this contribution is only $\approx 8 \%$ of continuum at $\lambda 5870 \AA$. In the high state (see below) the same contribution increases to $\approx 20 \%$.

The grid computations in Tables $3(a)$ and $3(b)$ are visualized by adding star cluster spectra with ages and metallicities as near as possible as those in the path. We used $G 5, G 3$, and $G 1, I 2$, and $I 1, Y 4, Y 3$, and $Y 1$, and $R H$ II from Bica (1988). This procedure is illustrated in Fig. 4 for the 1985 low-state nuclear spectrum. The synthesis computations are reddening independent because they use only equivalent widths. An internal reddening estimation is possible com-
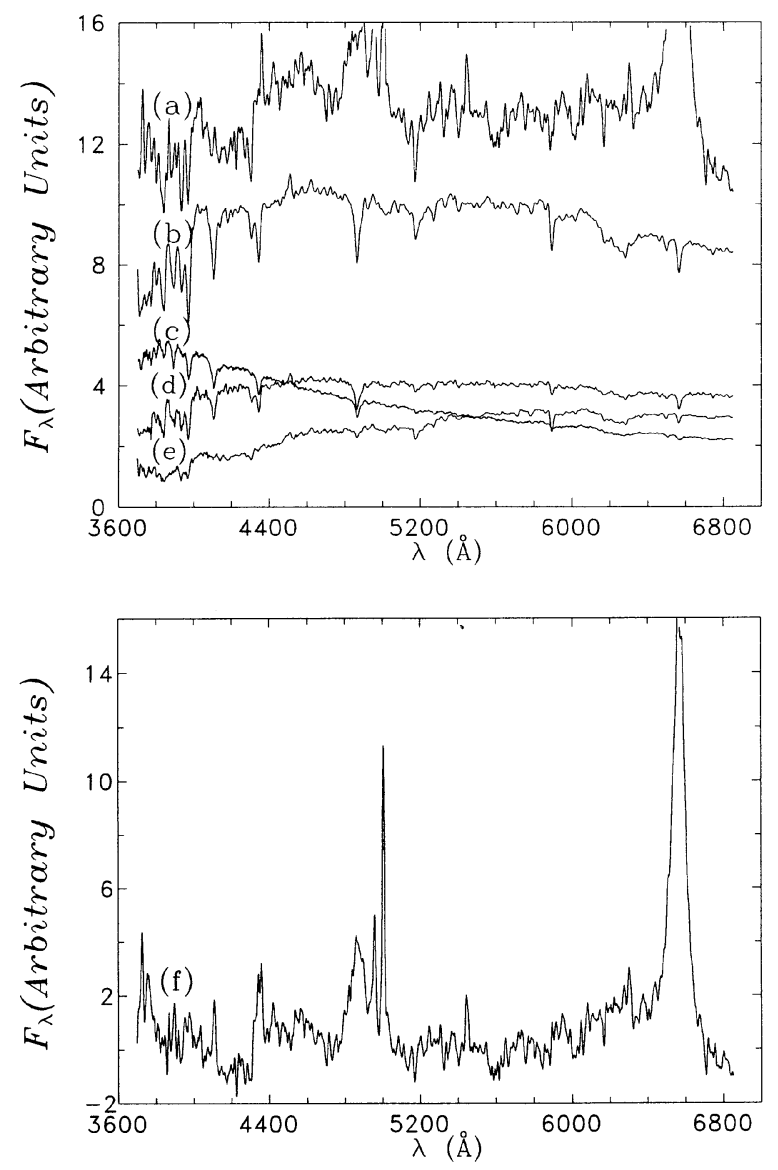

FIG. 4. Visualization of the stellar population synthesis. (a) The 1985 low-state nuclear spectrum; (b) The synthesized stellar population template; (c) Sum of the young components ( $Y 1, Y 3, Y 4, R \mathrm{H}$ II); (d) Sum of the intermediate age components $(I 1, I 2)$; (e) Sum of the old components $(G 1, G 3, G 5)$; and (f) The resulting stellar population subtracted spectrum. Ordinate in arbitrary units. 
paring the observed spectrum to the synthesized one. We obtain $\mathrm{E}(B-V)_{I}=0.40$, suggesting an important dust content affecting the stellar population in the nucleus. For the off-nuclear spectrum no internal reddening correction is necessary. It would be important to study this galaxy in the infrared to search for dust emission.

\subsection{The Neighboring Compact Object}

The absence of Doppler shifts in the absorption line spectrum of this object indicates that it is a foreground star. We have compared it with a series of stars in the Gunn \& Stryker (1983) library and found that its spectral type lies between a K7V (HD151288) and an MOV (GC 15A). The spectra superimpose very well once the compact object's spectrum is reddening corrected with $\mathrm{E}(B-V)=0.05$, which is consistent with the foreground excess adopted for the galaxy.

\section{THE EMISSION SPECTRA}

The intensity and FWHM of the emission lines in the stellar-free nuclear spectra have been measured fitting gaussians to the observed profiles and are listed in Table 4 . We have deconvolved the emission lines in the IPCS spectrum with a gaussian with FWHM $=4 \AA$. Below we comment on the main characteristics of each spectrum.

\subsection{The 1985 Low-State Spectrum}

We have found two components for $\mathrm{H} \alpha$ whereas only a broad component was found in $\mathrm{H} \beta$. The narrow component of $\mathrm{H} \alpha$ contributes with about $7 \%$ to the total $\mathrm{H} \alpha$ flux, and if we expect recombinations as the main mechanism for Balmer line formation, $\mathrm{H} \beta$ narrow should be undistinguishable from the noise. Other noticeable spectral features are $[\mathrm{FeV} \mathrm{II}]_{\lambda 6087}$, and several $\mathrm{Fe}$ II blends around $\lambda 4570$ and $\lambda 5200 \AA$. We have compared the $\mathrm{H} \beta /[\mathrm{O} \text { III }]_{\lambda 5007}$ ratio in our spectrum with that of Morris \& Ward (1988) taken a year before. We conclude that $\mathrm{H} \beta$ has decreased a factor 3 in this period. It is interesting to point out that in this galaxy Penfold (1979) detected variation in his $U$-band photome-

TABLE 4. Nuclear emission-line parameters.

\begin{tabular}{cccccc}
\hline \hline & & \multicolumn{2}{c}{ Markarian50(1985) } & \multicolumn{2}{c}{ Markarian50(1990) } \\
\cline { 6 - 7 } \cline { 5 - 6 } Line & $\lambda_{O}$ & FWHM & Flux & FWHM & Flux \\
\hline$[\mathrm{OII}]$ & 3727 & 1070 & $26.6 \pm 2.5$ & - & - \\
{$[\mathrm{FeV}]+\mathrm{HeII}$} & 3796 & - & - & 945 & $20.6 \pm 3.0$ \\
$\mathrm{H} \epsilon+[\mathrm{NeIII}]$ & 3968 & - & - & 3110 & $118.4 \pm 10.0$ \\
$\mathrm{H} \delta$ & 4101 & 1240 & $26.4 \pm 1.2$ & 4210 & $229.4 \pm 14.0$ \\
$\mathrm{H} \gamma$ & 4340 & 1330 & $24.2 \pm 1.8$ & 3785 & $334.4 \pm 20.0$ \\
{$[\mathrm{OIII}]$} & 4363 & 1323 & $32.5 \pm 1.8$ & - & - \\
$\mathrm{HeII}$ & 4686 & - & - & 1750 & $20.5 \pm 2.2$ \\
$(\mathrm{H} \beta)_{B}$ & 4861 & 4500 & $202.3 \pm 15.0$ & 5350 & $666.1 \pm 35.0$ \\
$(\mathrm{H} \beta)_{N}$ & 4861 & - & - & 1600 & $105.1 \pm 10.0$ \\
{$[\mathrm{OIII}]$} & 4959 & 917 & $34.3 \pm 3.0$ & 1350 & $34.3 \pm 3.0$ \\
{$[\mathrm{OIII}]$} & 5007 & 908 & $104.1 \pm 3.0$ & 1335 & $104.1 \pm 8.5$ \\
$\mathrm{HeI}$ & 5876 & - & - & 6320 & $168.5 \pm 15.0$ \\
{$[\mathrm{FeVII}]$} & 6087 & 1140 & $26.9 \pm 8.0$ & 623 & $9.5 \pm 1.0$ \\
{$[\mathrm{OI}]$} & 6300 & 830 & $21.2 \pm 3.0$ & - & - \\
{$[\mathrm{NII}]$} & 6548 & 1372 & $34.9 \pm 5.0$ & 1870 & $110.4 \pm 20.0$ \\
$(\mathrm{H} \alpha)_{B}$ & 6563 & 4510 & $577.0 \pm 36.0$ & 5360 & $1347.0 \pm 90.0$ \\
$(\mathrm{H} \alpha)_{N}$ & 6563 & 639 & $38.9 \pm 5.0$ & 1592 & $390.0 \pm 16.0$ \\
{$[\mathrm{NII}]$} & 6584 & 1364 & $104.6 \pm 5.0$ & 1860 & $331.6 \pm 20.0$ \\
\hline \hline
\end{tabular}

Notes to TABLE 4

Line measurements after stellar population subtraction; B and $\mathrm{N}$ refer respectively to the broad and narrow components; The lines have been deconvolved with a gaussian with FWHM $=4 \AA$; Fluxes in $10^{-16} \mathrm{erg} \mathrm{cm}^{-2} \mathrm{~s}^{-1}$; FWHM in $\mathrm{km} \mathrm{s}^{-1}$; Errors in line fluxes are estimated according to the noise level around each line. try larger than $0.8 \mathrm{mag}$. The analyses above indicate that in June 1985 [Fig. 3(a)] Mk50 was in a quiet phase of activity, as denoted by the prominent stellar population and the weakness of the broad permitted lines and the almost absence of a non-stellar continuum.

\subsection{The 1990 High-State Spectrum}

The 1990 spectrum shows a remarkable development of the $\mathbf{H}$ I Balmer lines: $\mathbf{H} \gamma$ and $\mathbf{H} \boldsymbol{\delta}$ are strong and broad ( $F W H M>3700 \mathrm{~km} \mathrm{~s}^{-1}$ ), and $\mathrm{H} \beta$ almost completely drowns $[\mathrm{O} \mathrm{III}]_{\lambda 4959}$. Also present is a broad $\left(\mathrm{FWHM} \approx 6300 \mathrm{~km} \mathrm{~s}^{-1}\right.$ ) $\mathrm{He}_{\lambda 5876}$. The continuum presents a steep rise blueward of $\lambda 4000 \AA$ characteristic of the UV bump seen in most Seyfert 1's.

The stellar population contribution in this high state level was estimated as follows. We have scaled the [O III] lines between the high and low state spectra, because narrow line components are not expected to vary significantly in only 5 yr [Fig. 3(a)]. Taking as reference the synthetic stellar population spectrum for the low-state (Sec. 4.1) we conclude that the stellar component is responsible for $48 \%$ at $\lambda 3800$ $\AA, 71 \%$ at $\lambda 5000 \AA$, and $82 \%$ at $\lambda 8900 \AA$ of the observed continuum.

After subtraction of the stellar population we made the measurements of the emission lines as in Sec. 5.1 and the results are listed in Table 4.

The variation occurred between 1985 and 1990 is best visualized in the difference spectrum (1990-1985) shown in Fig. 5. The main features of this spectrum are the prominent $\mathrm{H}$ I lines, strong $\mathrm{He}_{\lambda 5876}$ and $\mathrm{He}_{\mathrm{II}_{\lambda 4686}}$, and the apparently complete disappearance of forbidden lines. An excess is observed for $\lambda<4000 \AA$, and the Fe II lines are completely cancelled out by the subtraction. In Fig. 3(b) we show in more detail the region where $\mathrm{Fe}$ II lines are in general strongest in Seyferts and QSOs (Phillips 1978). This spectral region, which corresponds to the Fe II multiplets 37,38 , and 43, is very similar in Mk50 to those in the objects in his Fig. 3. The high and low-state spectra are plotted in scale as in Fig. 3 (a) (normalized to the same [O III $]_{\lambda 4959,5007}$ flux), only a constant was added to the low-state spectrum for a better visualization. We conclude that the Fe II is very similar in

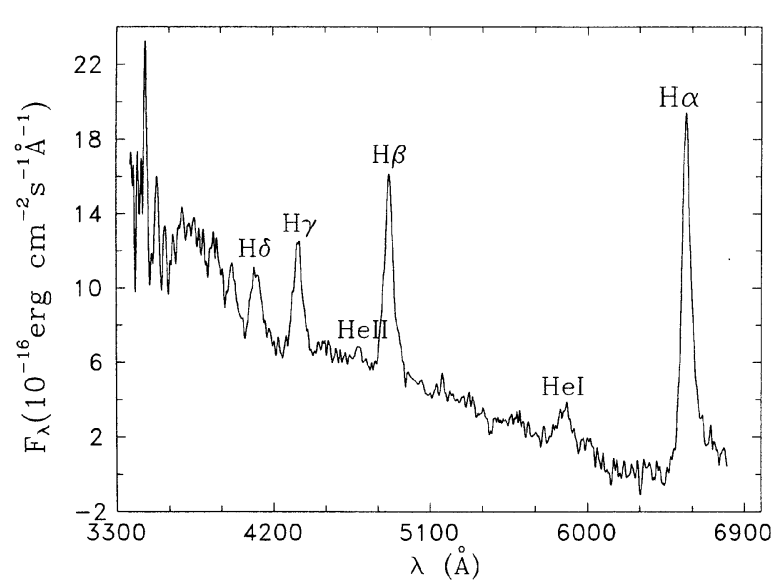

Fig. 5. The difference of the 1990 high state and the 1985 low-state spectra. Forbidden lines and Fe II lines are cancelled out. Ordinate units as in Fig. 3. 
TABLE 5. Off-nuclear emission-line parameters.

\begin{tabular}{cccc}
\hline Line & $\lambda_{O}$ & FWHM & Flux \\
\hline$[\mathrm{OII}]$ & 3727 & 890 & $16.2 \pm 5.0$ \\
$\mathrm{H} \beta$ & 4861 & 1110 & $9.3 \pm 1.0$ \\
{$[\mathrm{OIII}]$} & 4959 & 1300 & $3.1 \pm 0.3$ \\
{$[\mathrm{OIII}]$} & 5007 & 1300 & $9.3 \pm 0.3$ \\
{$[\mathrm{NII}]$} & 6548 & 1120 & $8.9 \pm 1.0$ \\
$\mathrm{H} \alpha$ & 6563 & 1120 & $19.7 \pm 1.0$ \\
{$[\mathrm{NII}]$} & 6584 & 1120 & $26.7 \pm 1.0$ \\
{$[\mathrm{SII}]$} & $6717+6731$ & 1265 & $13.7 \pm 1.1$ \\
\hline \hline
\end{tabular}

Notes to TABLE 5

Fluxes measured on the off-nuclear average spectrum, after the stellar population subtraction; units as in Table 4.

both cases, the only difference in the region appears to be due to the presence of $\mathrm{He}_{\lambda 4471}$ and $\mathrm{He} \mathrm{II}_{\lambda 4686}$ in the high-state spectrum. This suggests that in $\mathrm{Mk} 50, \mathrm{Fe}$ II originates closer to the narrow line region rather than in the broad line region. It would be interesting to observe $\mathrm{Mk50}$ in higher dispersion in order to check the profiles of individual Fe II lines.

The change in the $\mathrm{H} \alpha$ luminosity amounts to $\Delta L(\mathrm{H} \alpha)=(5.36 \pm 0.5) \times 10^{39} \mathrm{ergs} \mathrm{s}^{-1}$.

\subsection{The Off-Nuclear Spectrum}

Emission lines have also been detected in the off-nuclear spectrum $(3.5<R<6.5 \mathrm{kpc})$. After subtraction of the appropriate template for the stellar population we measured the emission line fluxes which are listed in Table 5. The emission lines in the subtracted spectrum resemble those of an AGN rather than a typical $\mathrm{H}$ II region. In fact, line-ratios plotted in the BPT diagrams (Baldwin et al. 1981) fall in the region of spectra ionized by a power law. Although there is evidence of a rather old burst of star formation $\left(\approx 10^{8} \mathrm{yr}\right.$,
Sec. 3.2) the gas in this region seems to be ionized mostly by the nuclear source.

\section{CONCLUSIONS}

The main conclusions of this work can be summarized as follows:

(a) The $V$ and $I$ luminosity profiles are fitted by the $R^{1 / 4}$ law; there is no evidence of a disk structure; the underlying stellar population has a $(V-I)$ color index similar to that of an elliptical or S0 nucleus; and there is a small contribution of blue light, possibly from a burst of star formation of age approximately $10^{8} \mathrm{yr}$; the star formation is strong enough to be detected in the $(V-I)$ color, but not to destroy the overall de Vaucouleurs profiles.

(b) As for the underlying stellar population, we found that the stellar component is typical of a red strong-lined bulge population with a contribution from young components (age $<5 \times 10^{8} \mathrm{yr}$ ) of $\approx 8 \%$ at $\lambda 5800 \AA$. This red stellar population is dominant out to $8 \mathrm{kpc}$.

(c) In the period 1985-1990 Mk50 changed remarkably its nuclear spectrum; $\mathrm{H} \beta$ becomes a factor $\approx 7$ stronger than [O III $]_{\lambda 5007}$, and Helium lines develop notably; the change in the $\mathrm{H} \alpha$ luminosity amounts to $\approx 5 \times 10^{39} \mathrm{ergs} \mathrm{s}^{-1}$.

(d) $\mathrm{Mk50}$ has a ratio mass of neutral gas to blue luminosity $\left(\mathscr{M}_{H} / L_{B}\right)$ of 0.11 (Mirabel \& Wilson 1984). This is larger than the ratio observed in typical elliptical galaxies, which the above authors found to be 0.01 . On the other hand the $V$ and $I$ luminosity profiles, integrated color index $(V-I)$, and the stellar population are all consistent with those of an elliptical galaxy with some recent star formation superimposed.

We have also shown that a point source near to the nucleus is in fact a foreground star, of spectral type between K7V and MOV.

This work has been partially supported by the Brazilian institution $\mathrm{CNPq}$.

\section{REFERENCES}

Adams, T. F. 1977, ApJS, 33, 19

Baldwin, J. A., Phillips, E. M., and Terlevich, R. 1981, PASP, 551, 5

Bica, E. 1988, A\&A, 195, 76

Bica, E., Alloin, D., and Schmidt, A. 1990, MNRAS, 242, 241

Boronson, T. 1981, ApJS, 46, 177

Burstein, D., and Heiles, C. 1984, ApJS, 54, 33

Dahari, O. 1984, AJ, 89, 966

de Vaucouleurs, G., de Vaucouleurs, A., and Corwin, H. G. 1976, Second Reference Catalogue of Bright Galaxies (University of Texas Press, Austin)

Freeman, K. C. 1970, ApJ, 160, 811

Gunn, J. E., and Stryker, L. L. 1983, ApJS, 52, 121

Kim, D. W. 1989, ApJ, 346, 653

Landolt, L. 1983, AJ, 88, 439

Mediavilla, E. M., Pastoriza, M. G., and Battaner, E. 1989, Ap\&SS, 157, 145
Mirabel, I. F., and Wilson, A. S. 1984, ApJ, 277, 92

Morris, S. L., and Ward, M. J. 1988, MNRAS, 230, 639

Osterbrock, D. 1988, Astrophysics of Gaseous Nebulae and Active Galactic Nuclei (University Science Books, Mill Valley)

Penfold, J. E. 1979, MNRAS, 186, 297

Phillips, M. M. 1978, ApJS, 38, 187

Sandage, A., and Tammann, G. 1981, A Revised Shapely-Ames Catalogue of Bright Galaxies (Carnegie Institution of Washington, Washington, DC)

Sargent, W. L. W. 1970, ApJ, 159, 765

Simien, F., and de Vaucouleurs, G. 1986, ApJ, 302, 564

Weedman, D. W. 1976, ARA\&A, 21, 55

Wilson, A. S., and Meurs, E. J. A. 1982, A\&AS, 50, 217 